\title{
ELECTROSTATIC DOMINO ACTUATORS
}

\author{
Guo D. Su, Li Fan, and Ming C.Wu \\ Electrical Engineering Department, UCLA \\ 66-147D Engineering IV, 405 Hilgard Avenue, Los Angeles, CA 90095-1594 \\ TEL: 310-825-6859, EMAIL: wu@icsl.ucla.edu
}

\begin{abstract}
A surface-micromachined electrostatic domino actuator (EDA) has been proposed and successfully demonstrated. The EDA consists of $\mathrm{N}$ stages of gap-closing actuators with linearly increasing gap spacing. Large displacement and large force are achieved by the domino effect created by successive pull-in of the gap-closing actuator array. Experimentally, a displacement of 24 $\mu \mathrm{m}$ (bi-directional) at $35 \mathrm{~V}$ bias has been successfully achieved by a four-stage EDA.
\end{abstract}

\section{INTRODUCTION}

Electrostatic force is very attractive for micro-electromechanical systems (MEMS) because of good scaling properties to small dimensions, high energy densities, and ease of fabrication [1]. Gap-closing and comb drive actuators are two most popular electrostatic actuators $[2,3]$. The gap-closing actuators have large force, however, their displacement is limited because the electrostatic force is inversely proportional to the square of the gap separation. Recently, several approaches have been proposed to increase the displacement of gap-closing actuators [4-6]. $28 \mu \mathrm{m}$ displacement (at 160V) and $6.3 \mu \mathrm{N}$ (at 200V) force has been achieved in [4], however, it is difficult to realize the structure by surface-micromachining technology. The curved electrode actuator can achieve large displacement at low voltage ( $30 \mu \mathrm{m}$ at $40 \mathrm{~V})$ [6], however, the force is small at zero displacement and the flexible beam could deform when driving a load.

In this paper, we propose a new large-force, largedisplacement actuator, called electrostatic domino actuator (EDA). The domino actuator consists of an array of serially connected gapclosing actuators with linearly increasing gap sizes. The first gapclosing actuator with small gap separation determines the force of the EDA, while the last gap-closing actuator with large gap spacing determines the displacement of EDA. Experimentally, a four-stage EDA has been successfully demonstrated using the polysilicon surface-micromachining technology. Bi-directional displacement of $24 \mu \mathrm{m}$ has been achieved at a bias voltage of 35 V. The force at zero displacement is $8.5 \mu \mathrm{N}$. The experimental results agree very well with theory. The EDA can be easily integrated with other microstructures using the well-established surface micromachining fabrication technology.

\section{PRINCIPLE}

The EDA comprises an array of gap-closing actuators with linearly increasing gap separation. In contrast to the distributed electrostatic microactuators in Ref. [4], the stators (fixed electrode) of all gap-closing actuators are anchored to the substrate. The movers (movable electrode) are suspended from the substrate by springs and connected to each other by "pull-only" joints. The gap spacing increases linearly from the first to the final stages: it is $\Delta$ for the first stage, $2 \Delta$ for the second stage, and $n \Delta$ for the n-th stage. The scanning electron micrographs (SEM) of the EDA and the "pull-only" joints are shown in Fig. 1(a) and (b), respectively.

The EDA has two electrodes, one connects to all stators, and the other connects to all movers. The working principle of EDA is illustrated in Fig. 2. Initially, when a voltage applied, the first stage is most effective because of the small gap separation. When the first stage mover is pulled in, all the following stages are moved by the same displacement, $\Delta$, as illustrated in Fig. 2(b). Then the resulting gap spacing of second stage becomes $2 \Delta-\Delta=\Delta$, which is the same as that of the original spacing of the first stage. As the second gap-closing actuator pulls in, the remaining stages are moved by another $\Delta$, as shown in Fig. 2(c). The same process is repeated at every stage until the last stage was pulled in. The displacement of the last stage is $N \times \Delta$, where $N$ is the number of stages.

The EDA has the following unique features:

1) Large displacement (determined by the last gap-closing actuator),

2) Large force at zero displacement (determined by the first gapclosing actuator),

3) Low voltage operation,

4) The EDA can be easily integrated with other surfacemicromachined structures.

\section{FABRICATION}

The domino actuator is fabricated by the surface micromachining technology at MEMS Technology Application Center at North Carolina (MCNC). The two polysilicon layers are stacked to increase aspect ratio for larger force. The current design has four stages in both directions, as shown in Fig. 1(a). The electrodes are $3.5 \mu \mathrm{m}$ high and $1 \mathrm{~mm}$ long. The gap spacing is 3,6 , 9 , and $12 \mu \mathrm{m}$, respectively. The force of the domino actuators can be further increased by employing high-aspect-ratio electrodes such as deep reactive ion etched (DRIE) structures.

\section{RESULTS AND DISCUSSION}

The domino actuator has been successfully demonstrated. The displacement of EDA as a function of applied voltage has been measured. To determine pull-in voltage, the driving voltage was slowly increased until the mover at each stage hit the polysilicon stopper. The results are shown in Fig. 3. A maximum displacement of $24 \mu \mathrm{m}(12 \mu \mathrm{m}$ in each direction) has been achieved at a bias voltage of $35 \mathrm{~V}$. The experimental results agree very well with theoretical calculations, as shown in Fig. 3.

Compared with conventional gap closing actuators, the EDA offers larger displacement, larger force, and lower operating voltage. This can be understood by examining the expression for electrostatic force for parallel plates:

$$
F=-\frac{1}{2}-\frac{\varepsilon_{0} t l V^{2}}{d^{2}}
$$

where $\varepsilon_{0}$ is the dielectric constant, $t$ and $l$ are the thickness and length of the electrodes, respectively, $d$ is the gap spacing, and $V$ is the driving voltage. The electrostatic force is inversely proportional to the square of the gap spacing. The EDA effectively reduces the gap spacing by a factor of $\mathrm{N}$, where $\mathrm{N}$ is the number of stages. The resulting electrostatic force is $\mathrm{N}^{2}$ larger than that of the conventional gap closing actuator with the same total displacement. The displacement-vs-voltage characteristics of the conventional gap-closing actuator with the same displacement is 
also shown in Fig. 3. The pull-in voltage has been reduced from 46 $\mathrm{V}$ to $35 \mathrm{~V}$. Figure 4 shows the calculated forces of the EDA and the gap-closing actuators at $100 \mathrm{~V}$ bias. The electrostatic force at zero displacement has been increased from 2.3 to $8.5 \mu \mathrm{N}$.

The performance of the EDA can be further optimized. The current design employs the same springs for all stages. By properly choosing the individual spring constants, the EDA performance can be further optimized. Our theoretical calculation shows that a bi-directional displacement of $50 \mu \mathrm{m}$ can be achieved at $32 \mathrm{~V}$ bias with an optimized a 5-stage EDA.

\section{CONCLUSION}

A novel electrostatic domino actuator (EDA) has been proposed and successfully demonstrated to increase the displacement of gap-closing actuators. The domino effect in the EDA increases its total displacement while maintaining the force. Experimentally, a displacement of $24 \mu \mathrm{m}$ has been achieved at a applied voltage of $35 \mathrm{~V}$. The force at zero displacement is calculated to be $8.5 \mu \mathrm{N}$. The actuator performance can be further optimized.

\section{REFERENCES}

1. W. Trimmer and R. Jebens, “Actuators for Micro Robots ", Proceedings. 1989 IEEE International Conference on Robotics and Automation (Cat. No.89CH2750-8), 1989, pp. 1547-1552

2. W. Trimmer and R. Jebens, "Design Consideration for a Practical Electrostatic Micro-motor", Sensors and Actuators, vol. 11, 1987, pp. 189-206

3. W. C. Tang, C. H. Nguyen, and R. T. Howe, "Laterally Driven Polysilicon Resonant Microstructures", Sensors and Actuators, vol. 20, 1990, pp. 25-32

4. K. Minami, S. Kawamura, and M. Esashi, "Fabrication of Distributed Electrostatic Micro Actuator", Journal of Microelectromechanical Systems, vol. 2, no. 3, September 1993, pp. 121-127

5. S. Bobbio, M. Kellam, B. Dudley, S. Johnson, S. Jones, J. Jacobson, F. Tranjan, and T Dubois, "Integrated Force Arrays ", in Proc. IEEEE Micro Electro Mechanical Syst. Workshop, 1993, pp. 149-154

6. R. Legtenberg, E. Berenschot, M. Elwenspoek, and J. Fluitman, "Electrostatic Curved Electrode Actuators ", Proceedings. IEEE Micro Electro Mechanical Systems 1995, pp. 37-42

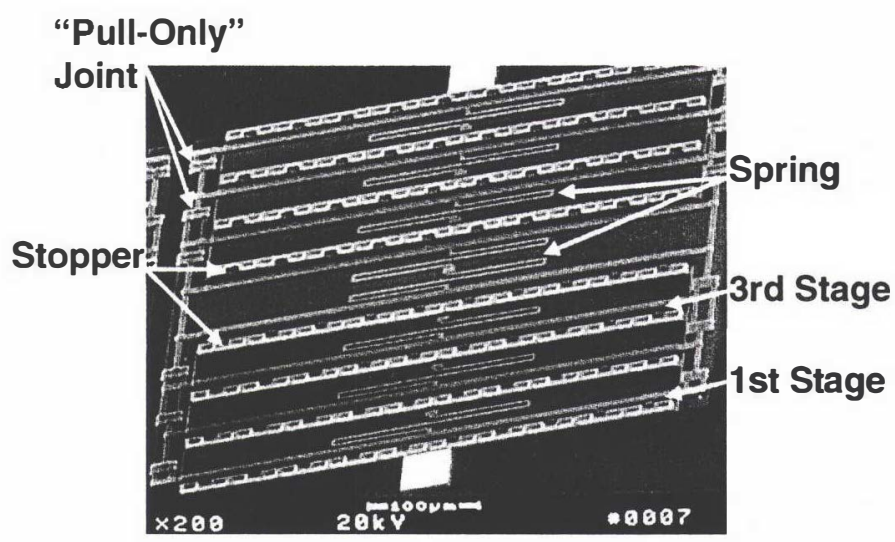

(a)

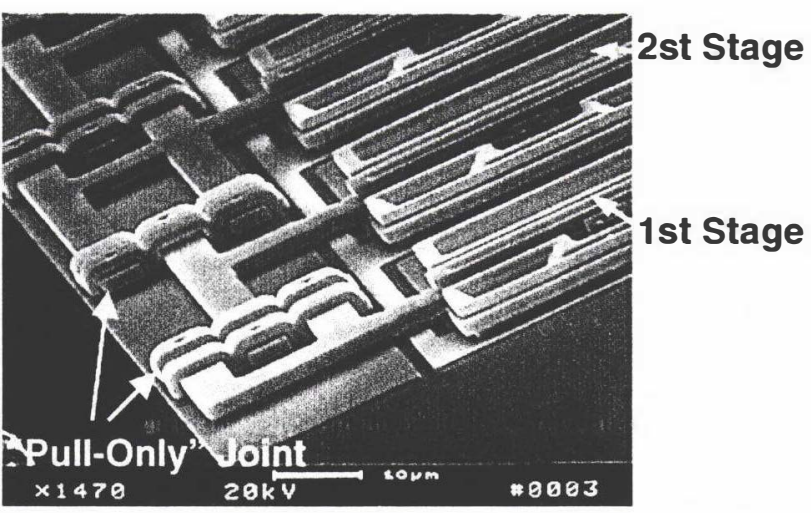

(b)

Figure1. (a) SEM micrograph of electrostatic domino actuator (EDA). (b)Close-up look of the "pull-only" joint.

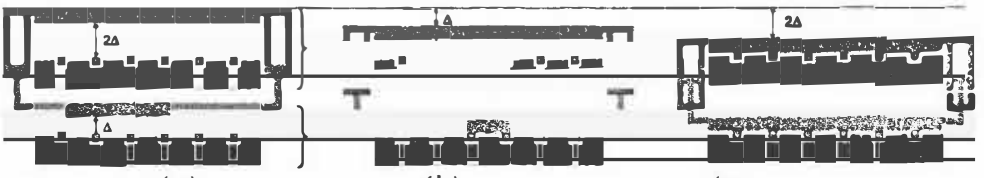

(a)

(b)

(c)

Figure2. Principle of electrostatic domino actuator (only 2 stages are shown here).
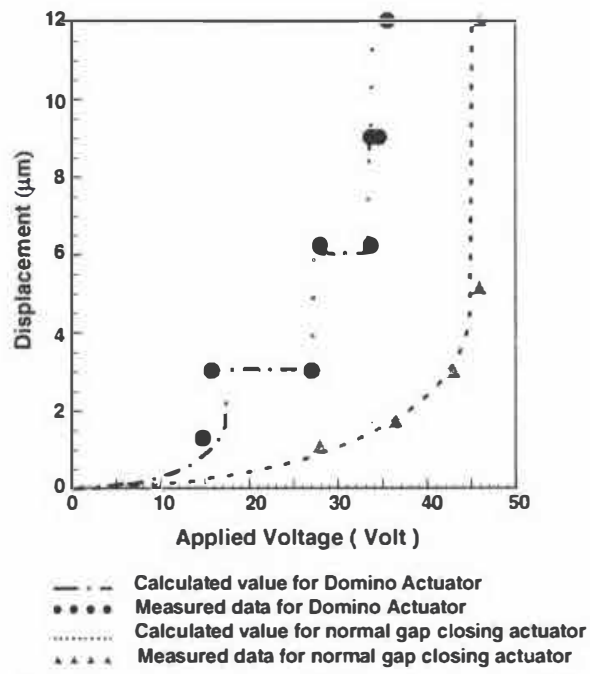

Figure 3. Displacement vs. applied voltage for EDA and gap closing actuators.

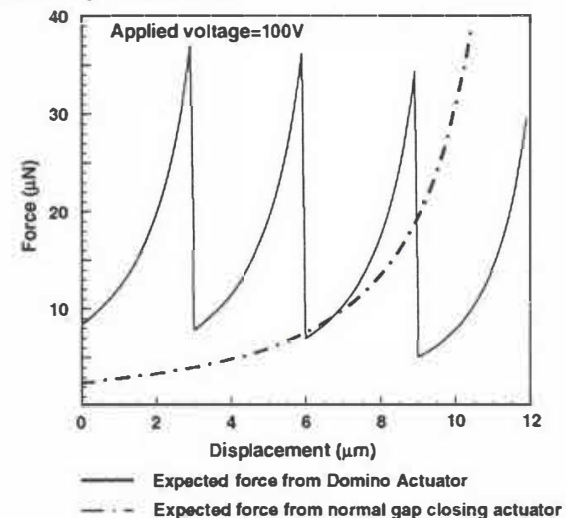

Figure 4. Displacement vs. forces generated by EDA and gap closing actuators. 